\title{
Interpatient heterogeneity in hepatic microvascular blood flow during vascular inflow occlusion (Pringle manoeuvre)
}

\author{
Lucinda Shen ${ }^{1,2 \#}$, Zühre Uz ${ }^{1,3 \#}$, Joanne Verheij ${ }^{4}$, Denise P. Veelo ${ }^{5}$, Yasin Ince ${ }^{1}$, Can Ince ${ }^{1,2}$, \\ Thomas M. van Gulik ${ }^{3}$
}

${ }^{1}$ Department of Translational Physiology, Amsterdam UMC, Location Academic Medical Center, University of Amsterdam, Amsterdam, The Netherlands; ${ }^{2}$ Department of Intensive Care, Laboratory of Translational Intensive Care, Erasmus MC, University Medical Center, Rotterdam, The Netherlands; ${ }^{3}$ Department of Surgery, ${ }^{4}$ Department of Pathology, ${ }^{5}$ Department of Anesthesiology, Amsterdam UMC, Location Academic Medical Center, University of Amsterdam, Amsterdam, The Netherlands

Contributions: (I) Conception and design: L Shen, Z Uz, J Verheij, DP Veelo, C Ince, TM van Gulik; (II) Administrative support: L Shen, Z Uz, J Verheij; (III) Provision of study materials or patients: DP Veelo, C Ince, TM van Gulik; (IV) Collection and assembly of data: L Shen, Z Uz, TM van Gulik; (V) Data analysis and interpretation: L Shen, Z Uz, J Verheij, DP Veelo, C Ince, TM van Gulik; (VI) Manuscript writing: All authors; (VII) Final approval of manuscript: All authors.

\#These authors contributed equally to this work.

Correspondence to: Prof. Dr. Thomas M. van Gulik, MD. Department of Surgery, Amsterdam UMC, University of Amsterdam, Meibergdreef 9, 1105 AZ, Amsterdam, The Netherlands. Email: t.m.vangulik@amsterdamumc.nl.

Background: Vascular inflow occlusion (VIO) during liver resections (Pringle manoeuvre) can be applied to reduce blood loss, however may at the same time, give rise to ischemia-reperfusion injury (IRI). The aim of this study was to assess the characteristics of hepatic microvascular perfusion during VIO in patients undergoing major liver resection.

Methods: Assessment of hepatic microcirculation was performed using a handheld vital microscope (HVM) at the beginning of surgery, end of VIO (20 minutes) and during reperfusion after the termination of VIO. The microcirculatory parameters assessed were: functional capillary density (FCD), microvascular flow index (MFI) and sinusoidal diameter (SinD).

Results: A total of 15 patients underwent VIO; 8 patients showed hepatic microvascular perfusion despite VIO (partial responders) and 7 patients showed complete cessation of hepatic microvascular perfusion (full responders). Functional microvascular parameters and blood flow levels were significantly higher in the partial responders when compared to the full responders during VIO (FCD: $0.84 \pm 0.88 v s$.

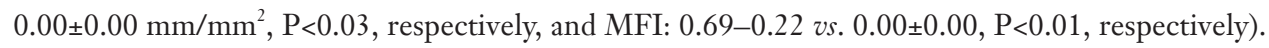

Conclusions: An interpatient heterogeneous response in hepatic microvascular blood flow was observed upon VIO. This may explain why clinical strategies to protect the liver against IRI lacked consistency.

Keywords: Liver; perfusion; microcirculation

Submitted Nov 22, 2019. Accepted for publication Feb 06, 2020.

doi: $10.21037 / \mathrm{hbsn} .2020 .02 .04$

View this article at: http://dx.doi.org/10.21037/hbsn.2020.02.04 


\section{Introduction}

Blood loss during hepatic resections is a major variable influencing postoperative outcomes (1). For safe resections, surgeons may exercise vascular control in order to reduce the need for blood transfusions (2). Vascular inflow occlusion (VIO) by the Pringle manoeuvre entails simultaneous clamping of the portal vein and the hepatic artery in the hepatoduodenal ligament (2).

This is a consistent method of vascular control which may be performed continuously or intermittently. Resections using intermittent VIO involve 15-20 minutes on clamp followed by $5-10$ minutes off clamp. Studies have shown that intermittent VIO can be safely applied for up to 120 minutes, granting the surgeon a longer cumulative occlusion time when faced with a more complex tumour presentation $(3,4)$.

A potential consequence of intermittent VIO is the induction of ischemia-reperfusion injury (IRI) resulting in hepatocellular and sinusoidal endothelial cell (SEC) injury $(5,6)$. Occlusion of the hepatoduodenal ligament using a tourniquet suspends blood flow to the liver parenchyma, causing cellular oxygen deprivation. The stressed hepatocytes switch to anaerobic respiration leading to an increase in lactic acid production and subsequent activation of apoptotic factors $(7,8)$. When VIO is terminated as the tourniquet is released, blood flow is restored to the hepatocytes. On the microcirculatory level, leukocytes are signalled for transmigration into the parenchyma through integrin interactions between the SECs and the leukocyte cell surface (9). Infiltration of leukocytes however is detrimental to the microcirculation as it may result in SEC death, vasoconstriction and sinusoidal shut down. Once inside the parenchyma, leukocytes undergo oxidative stress and production of reactive oxygen species $(10,11)$. Consequently, tissue damage of the future remnant liver (FRL) is incurred by the sterile inflammation which in turn, affect the FRL's ability to maintain function and to regenerate. Incompetent regenerative potential of the FRL increases the risk of post-hepatectomy liver failure (PHLF) $(12,13)$. Alterations in liver perfusion have been well studied in clinical settings such as liver resection, transplantation, tumour imaging and cirrhosis (14-18). However, microvascular changes during VIO are still poorly understood (19).

Introduction of handheld vital microscopes (HVMs) provides a non- invasive modality for the direct assessment and visualisation of the hepatic sinusoidal angioarchitecture. These devices have been applied intraoperatively to assess hepatic perfusion and microcirculatory alterations in a wide range of clinical settings such as transplantation, liver resection, portal vein embolization and mesenteric-portal bypass interpositioning (14,20-24). The data shows not only the feasibility of using HVMs intraoperatively to monitor changes in hepatic microcirculation, but also its applicability in a wide range of parenchymal diseases.

The current study aimed to use Cytocam incident dark field imaging (IDFI), which is the third and latest generation of HVMs, for the assessment of liver perfusion during VIO. Previous clinical data showed inconsistencies in the efficacy of strategies to protect the liver against IRI following VIO (25). Therefore, investigations of the liver microvascular blood flow states during and after VIO is important, as the effects of VIO during parenchymal transections may vary in terms of blood loss. To this end, the hepatic micro-angioarchitecture and quantitative microcirculatory parameters were examined in patients undergoing major liver resections using VIO.

\section{Methods}

\section{Study population}

This is a single-centre, prospective observational study evaluating the liver microcirculation using Cytocam-IDF imaging (Braedius Medical, Huizen, The Netherlands). Data was obtained of patients over the age of 18 diagnosed with primary or secondary liver cancer undergoing elective major liver resection at the Department of Surgery of Amsterdam UMC, Location AMC, Amsterdam, The Netherlands. Exclusion criteria for the study were: body mass index $>30 \mathrm{~kg} / \mathrm{m}^{2}$, presence of an aberrant left hepatic artery recognised on contrast enhanced CT or otherwise identified at the beginning of surgery, diagnosis of severe hepatic steatosis (grade $3 ;>60 \%$ ), or withdrawal of informed consent. The study was conducted in compliance with ethical principles and appropriate regulatory requirements. All procedures were carried out in concordance with the Declaration of Helsinki. Ethical consent was given by the ethical committee at the Amsterdam UMC (ethical committee reference number W17_259). All participants eligible for inclusion received full explanation of the 
protocol and provided informed consent at least 24 hours before surgery.

\section{Study design}

A baseline hepatic microcirculatory measurement was made at the beginning of surgery after laparotomy; on segment 3 in patients undergoing elective right hemi-hepatectomy and on segment 6 in elective left hemi-hepatectomy. A second microcirculatory measurement was made at the end VIO and a third microcirculatory measurement within 1-minute following termination of VIO, i.e., release of the tourniquet. For patients undergoing multiple rounds of VIO, hepatic microcirculation was only assessed during the first cycle of VIO.

\section{Hepatic VIO}

VIO was performed by a surgeon ( $\mathrm{TvG}$ ) experienced in liver surgery. After opening of the lesser omentum, a Mersilene tape was placed around the hepatoduodenal ligament and both ends pulled through a rubber tube for vascular occlusion. By pulling the tape tight through the tube and fixation with a clamp on the tube, blood flow from the hepatic artery and the portal vein was interrupted. Intermittent VIO was achieved using the tourniquet method for cycles of 20 minutes followed by 10 minutes of reperfusion during parenchymal transection.

\section{Hepatic microcirculatory imaging}

Intraoperative hepatic microcirculatory measurements were accomplished using Cytocam-IDF imaging. Cytocam is the third and latest generation of HVMs with factor 4 magnification and a large sensor image area, yielding a high-resolution field of view. With concentrically placed LED lights, Cytocam expels green light at $530 \mathrm{~nm}$ corresponding to the oxy-and deoxyhaemoglobin absorption range. The green light epi-illuminates the tissue surface causing haemoglobin to appear dark on the monitor screen. With a short illumination pulse time of $2 \mathrm{~ms}$, this enables tracking of the red blood cells (RBCs) and identification of perfused vessels. Vessels not containing RBCs will not be visible. Image sequences are projected onto a monitor which enables real-time identification of the liver sinusoidal micro- angioarchitecture. During hepatic microcirculatory measurements, prior to entering the operative field, a sterile cap was placed onto the Cytocam and covered with a laparoscopy camera cover (Camera Cover, Microtek Medical B.V., Zutphen, The Netherlands).

During image acquisition, excess blood on the liver was wiped off with a sterile gauze. The Cytocam was held perpendicular to the liver parenchyma taking care not to exert too much pressure. Image sequences were captured at 24 frames per second during a maximum of 4 seconds using CCTools 1.7.12 (Braedius Medical, Huizen, The Netherlands) and saved onto a powerful medical grade computer.

\section{Examination of systemic haemodynamics and laboratory parameters}

Haemodynamic parameters including heart rate, systolic and diastolic blood pressures, mean arterial pressure and peripheral oxygen saturation were acquired from the OR anaesthesia monitors (Edwards Life sciences, Irvine, California, USA) at the same time-points as hepatic microcirculatory measurements. Arterial blood gas assessments were performed at the beginning of surgery, during VIO/the termination of VIO and finally, at the end of surgery. Due to the proximity of the timepoints; end of VIO and termination of VIO, only one arterial blood gas assessment was performed. Parameters such as blood haemoglobin concentrations, oxygen saturation are important as green light is absorbed by haemoglobin and abnormal readings may interfere with microcirculatory measurements and provide reason for data exclusion. High glucose concentrations have been associated with increase in oxidative stress which may in turn result in endothelial dysfunction (26), which may result in aberrant microvascular density distributions.

\section{Determination of liver function}

Test parameters governing parenchymal damage [aspartate aminotransferase (AST), alanine aminotransferase (ALT), alkaline phosphatase (ALP) and glutamyl transpeptidase (GGT)] and liver function [total serum bilirubin (TSB) levels and prothrombin time (PT)] were investigated. A baseline assessment was made in all patients prior to surgery and followed up at post-operative days (PODs) 1, 3, 5 and 7. Blood was drawn from the venous lines into EDTA tubes 
(Beckton Dickens, Plymouth, UK) and run by the hospital laboratory.

\section{Histopathological examination of liver tissue}

A liver biopsy was taken at the beginning of surgery following laparotomy, to allow for microscopical assessment of the liver parenchyma as routine part of the surgical procedure. Histological assessment was performed by one experienced in-hospital pathologist (JV). The extent of liver fibrosis was determined descriptively; independent of underlying disease (if present): none, perisinusoidal or periportal, perisinusoidal and portal/periportal, bridging fibrosis involving less than $50 \%$ of the portal tracts and/or central veins, bridging fibrosis involving more than $50 \%$ of the portal tracts and/or central veins and cirrhosis.

\section{Image analysis and quantification of liver microcirculatory parameters}

Following image acquisition, image sequences were stabilized using CCTools 1.7.12 (Braedius Medical, Huizen, The Netherlands), saved and exported as AVI-DV files onto a separate external hard-drive. All analyses were performed offline using the semi-automated analysis software AVA 3.2 (Automated Vascular Analysis, Microvision, The Netherlands) by an independent investigator (LS) (27).

Parameters governing tissue convection and diffusion were analysed as according to the current 2018 consensus (28). Convection describes the flow behaviour of RBCs visible inside the micro vessels that may show continuous, sluggish, intermittent or no flow. Diffusion describes the density of micro vessels containing visible RBCs. The functional parameters assessed were; functional capillary density (FCD) $\mathrm{mm} / \mathrm{mm}^{2}$ and microvascular flow index (MFI) expressed in arbitrary units. FCD is considered the golden standard for assessment of the microcirculation and described the density of vessels with visible blood flow. Vessels were manually determined using the AVA 3.2 software and the flow behaviour of RBCs in the individual vessels were identified by multiplying the density of the vessels with the flow state, giving rise to FCD. The general flow state recorded in each video clip can be determined by the MFI; this index can be calculated by splitting the screen into quadrants. Each quadrant is given a predominate type of flow as dictated by $1=$ intermittent flow, 2 = sluggish flow, $3=$ continuous flow and $0=$ no flow. The final MFI is determined for the video clip by averaging the quadrant scores. Sinusoidal diameter
(SinD) was determined by randomly taking the diameter of 4 vessels (one in each MFI quadrant) and averaged.

\section{Assessment of microvascular flow during occlusion}

The percentage vessel surface $\left[\left(\mathrm{mm}^{2} / \mathrm{mm}^{2}\right) \times 100 \%\right]$ (PVS) is a parameter automatically calculated by the AVA 3.2 software following the drawing of individual vessels. The PVS was determined for all image sequences at the end of VIO of all the visible vessels (PVS all vessels). Then a new analysis window was generated of the same image sequence, and only the vessels with visible blood flow were manually drawn (PVS only perfused vessels). The percentage of perfused vessel surface (PPVS \%) during occlusion was calculated using the following formula:

$$
\text { PPVS\% }=\left(\frac{P V S \text { only perfused vessels }}{P V S \text { all vessels }}\right) \times 100 \%
$$

\section{Statistical analysis and data handling}

Statistical tests were performed using IBM SPSS statistics version 24.0 (BM Corp. Released 2016. IBM SPSS Statistics for Windows, Version 24.0. Armonk, NY, USA: IBM Corp). Graphs were produced using GraphPad Prism version 8 (GraphPad Software, La Jolla, California, USA).

All normally distributed data are displayed as means \pm standard deviation. To determine normal distribution a Shapiro-Wilks test was performed. An average of three analysed video clips was determined for baseline values. A one-way repeated measures ANOVA was performed to determine significance within the same group at the three different timepoints. An independent $t$-test was performed to determine significance between two different groups measuring the same parameter.

\section{Results}

\section{Patient characteristics}

A total of 15 patients were included in the study. The main demographics of these patients including their comorbidities and pathologies are shown in Table 1. A heterogeneity in microvascular sinusoidal blood flow of the liver was noted during VIO, hence the study population was partitioned into two groups for analysis; patients with no microvascular blood flow observed during VIO (full responders) $\mathrm{n}=7$ (Video 1), 
Table 1 Patient characteristics, comorbidity, surgical characteristics and hepatic pathology

\begin{tabular}{|c|c|c|c|}
\hline Parameters & $\begin{array}{c}\text { Full } \\
\text { responders, } \\
n=7\end{array}$ & $\begin{array}{c}\text { Partial } \\
\text { responders, } \\
n=8\end{array}$ & $P$ value \\
\hline
\end{tabular}

$\begin{array}{lccc}\text { Patient characteristics } & & & \\ \text { Gender (male: female) } & 4: 3 & 6: 2 & 0.847 \\ \text { Age (years) } & 70.1 \pm 6.4 & 65.1 \pm 10.4 & 0.290 \\ \text { BMl }\left(\mathrm{kg} / \mathrm{m}^{2}\right) & 24.4 \pm 3.6 & 24.1 \pm 3.0 & 0.883\end{array}$

Comorbidities

COPD $(n / n)$
Smoker $(n / n)$
Diabetes $(n / n)$
Hypertension $(n / n)$
Hypercholesterolemia $(n / n)$
Hepatic pathology
CGC $(n / n)$
$\operatorname{HCC}(n / n)$
$\operatorname{CRLM~}(n / n)$

Grade of fibrosis

$\begin{array}{lll}\text { No fibrosis } & 3 & 3 \\ \text { Perisinusoidal or periportal } & 1 & 1 \\ \begin{array}{l}\text { Perisinusoidal and portal/ } \\ \text { periportal }\end{array} & 1 & 1 \\ \begin{array}{l}\text { Bridging fibrosis, involving } \\ \text { less than } 50 \% \text { of the portal }\end{array} & 1 & 1 \\ \text { tracts and/or central veins } & \\ \begin{array}{l}\text { Bridging fibrosis, involving } \\ \text { more than } 50 \% \text { of the } \\ \text { portal tracts and/or central } \\ \text { veins }\end{array} & 0 & 1 \\ \text { Cirrhosis } & 1 & 1 \\ \end{array}$

BMI, body mean index; COPD, chronic obstructive pulmonary disease; CGC, cholangiocarcinoma; HCC, hepatocellular carcinoma; CRLM, colorectal liver metastasis. All data presented as mean \pm standard deviation.

and patients with persistent, partial microvascular blood flow despite VIO (partial responders) $n=8$ (Video 2). Clips of image sequences at sequential timepoints during the study of a full responder and a partial responder can be seen in the supplementary material.

\section{Intraoperative results}

The type of liver resection, duration of surgery and the cumulative VIO time is shown in Table 2. All patients received on average 2 cycles of VIO, the full responders had an average occlusion time of 42.6 minutes (95\% CI, 31.353.8 minutes) and the partial responders had an average occlusion time of 40.3 minutes (95\% CI, 24-56 minutes).

\section{Biochemical evaluation of arterial blood gas samples}

Arterial blood gas results can be seen in Table 3, no significant alternations in haemoglobin concentrations, $\mathrm{pH}$ and oxyhaemoglobin concentrations were detected across study timepoints. A steady increase in lactate concentrations was observed in both groups of patients from the beginning of surgery until the end of surgery. Although a significant increase in lactate concentrations were detected between groups at the end of VIO/termination of VIO, this significant difference did not persist at the end of surgery.

\section{Systemic bemodynamic evaluation}

At the systemic level, application of VIO was accompanied by a significant decrease in systolic blood pressure and mean arterial pressure when compared to the baseline in the full responders BP systolic; $88.71 \pm 21.09$ vs. $111.1 \pm 18.27$, $\mathrm{P}<0.006$, respectively. MAP; $62.14 \pm 10.33$ vs. $72.29 \pm 7.59$, $\mathrm{P}<0.03$, respectively (Figure 1). These values subsequently returned to baseline following termination of VIO after release of the tourniquet. All the while, blood pressure assessments remained relatively stable across timepoints in the partial responders. In addition, termination of VIO was accompanied by a significant decrease in heart rate of the partial responders (end of VIO $77.38 \pm 13.31 \mathrm{vs}$. termination of VIO $70.25 \pm 14.16$; $\mathrm{P}<0.01$ ) whereas the full responders showed no significant alterations in heart rate at the respective timepoints.

\section{Liver microcirculatory parameters}

The hepatic microcirculation parameters may be seen in Figure 2. At the end of VIO, the FCD and MFI (Figure $2 A, C$ respectively) were significantly higher in the partial 
Table 2 Surgical characteristics

\begin{tabular}{|c|c|c|c|}
\hline Parameters & $\begin{array}{l}\text { Full responders, } \\
\qquad \mathrm{n}=7\end{array}$ & $\begin{array}{c}\text { Partial } \\
\text { responders, } \\
n=8\end{array}$ & $P$ value \\
\hline \multicolumn{4}{|l|}{$\begin{array}{l}\text { Surgical } \\
\text { characteristics }\end{array}$} \\
\hline $\begin{array}{l}\text { Right hemi- } \\
\text { hepatectomy }(n / n)\end{array}$ & $4 / 7$ & $6 / 8$ & \\
\hline $\begin{array}{l}\text { Left hemi- } \\
\text { hepatectomy }(n / n)\end{array}$ & $3 / 7$ & $2 / 8$ & \\
\hline $\begin{array}{l}\text { Number of VIO } \\
\text { cycles }(n)\end{array}$ & $1.8 \pm 0.3$ & $1.9 \pm 0.6$ & 0.945 \\
\hline $\begin{array}{l}\text { Total occlusion time } \\
\text { (minutes) }\end{array}$ & $42.6 \pm 12.2$ & $40.2 \pm 18.9$ & 0.785 \\
\hline Blood loss (mLs) & $1,449.1 \pm 888.7$ & $1,669 \pm 1,146.9$ & 0.688 \\
\hline \multicolumn{4}{|l|}{$\begin{array}{l}\text { Number of segments } \\
\text { resected }(n)\end{array}$} \\
\hline One segment & 0 & 0 & \\
\hline Two segments & 0 & 0 & \\
\hline Three segments & 1 & 2 & \\
\hline Four segments & 4 & 3 & \\
\hline Five segments & 1 & 2 & \\
\hline Six segments & 1 & 1 & \\
\hline
\end{tabular}

responders showing sustained microvascular blood flow when compared to the full responders (no microvascular blood flow) (FCD $0.84 \pm 0.88$ vs. $0.00 \pm 0.00 \mathrm{~mm} / \mathrm{mm}^{2}$, $\mathrm{P}<0.03$, respectively and MFI $0.69 \pm 0.22$ vs. $0.00 \pm 0.00$, $\mathrm{P}<0.01$, respectively). Calculation of PPVS observed in the field of view of partial responders during analysis showed $9.33 \% \pm 4.62 \%$ (Figure $2 D$ ) at the time of VIO relative to the full responders which showed PPVS of $0 \%$ $(\mathrm{P}<0.0001)$. No significant differences in FCD and MFI were found between the groups at any other timepoint, and no significant differences were seen between groups in SinD (Figure 2B). Termination of VIO led to restoration of hepatic microvascular blood flow and caused a considerable increase in SinD when compared to the beginning of surgery in both the full responders and partial responders $(8.96 \pm 1.02$ vs. $11.83 \pm 1.92 \mu \mathrm{m}, \mathrm{P}<0.02$ and $8.34 \pm 0.53$ vs. $11.35 \pm 1.94 \mu \mathrm{m}, \mathrm{P}<0.02$ respectively).

\section{Liver function enzymes}

The results of liver function tests measured pre- and post-operatively are shown in Figure 3. Pre-operatively, ALT and ALP levels were higher in the partialresponders when compared to the full-responders (ALT: $60.29 \pm 59.3$ vs. $30.50 \pm 9.09$ IU/L, $\mathrm{P}<0.05$, respectively) and (ALP: $370.75 \pm 271.41$ vs. $142.8 \pm 62.95$ IU/L, $\mathrm{P}<0.01$,

Table 3 Arterial blood gas assessment

\begin{tabular}{|c|c|c|c|c|c|c|}
\hline $\begin{array}{l}\text { Parameters } \\
\text { Arterial blood gas } \\
\text { parameters }\end{array}$ & \multicolumn{4}{|c|}{ Full responder, $\mathrm{n}=7$} & \multicolumn{2}{|c|}{ Partial responder, $n=8$} \\
\hline $\mathrm{Hb}(\mathrm{mmol} / \mathrm{L})$ & $8.5 \pm 0.7$ & 7.50 .9 & $7.4 \pm 0.8$ & $8.0 \pm 1.3$ & $7.3 \pm 0.9$ & $7.5 \pm 1.3$ \\
\hline $\mathrm{pH}$ & $7.3 \pm 0$ & $7.4 \pm 0.1$ & 7.40 .1 & $7.4 \pm 0$ & $7.4 \pm 0.1^{*}$ & $7.4 \pm 0.1$ \\
\hline Glucose (mmol/L) & $8.5 \pm 3.3$ & $11.5 \pm 3.2^{\star}$ & $10.3 \pm 1.8$ & $7.7 \pm 1.4$ & $10.4 \pm 2.8$ & 9.031 .9 \\
\hline Lactate (mmol/L) & $1.0 \pm 0.3^{\#}$ & $2.9 \pm 1.7$ & $3.3 \pm 1.6^{\star \star}$ & $1.8 \pm 0.7$ & $3.3 \pm 1.0^{\star}$ & $3.4 \pm 0.8^{\star \star}$ \\
\hline
\end{tabular}

$\mathrm{Hb}$, haemoglobin; $\mathrm{HbO}_{2}$, oxyhaemoglobin; $\mathrm{VIO}$, vascular inflow occlusion; ${ }^{*}=$ within group one-way ANOVA analysis: ${ }^{*}, \mathrm{P}<0.05$ beginning of surgery vs. end of $\mathrm{VIO} \&$ termination of $\mathrm{VIO} ;{ }^{* *}, \mathrm{P}<0.05$ beginning of surgery vs. end of surgery. ${ }^{*}=$ between group unpaired $t$-test: \#, $\mathrm{P}<0.05$ full responders vs. partial responders. 

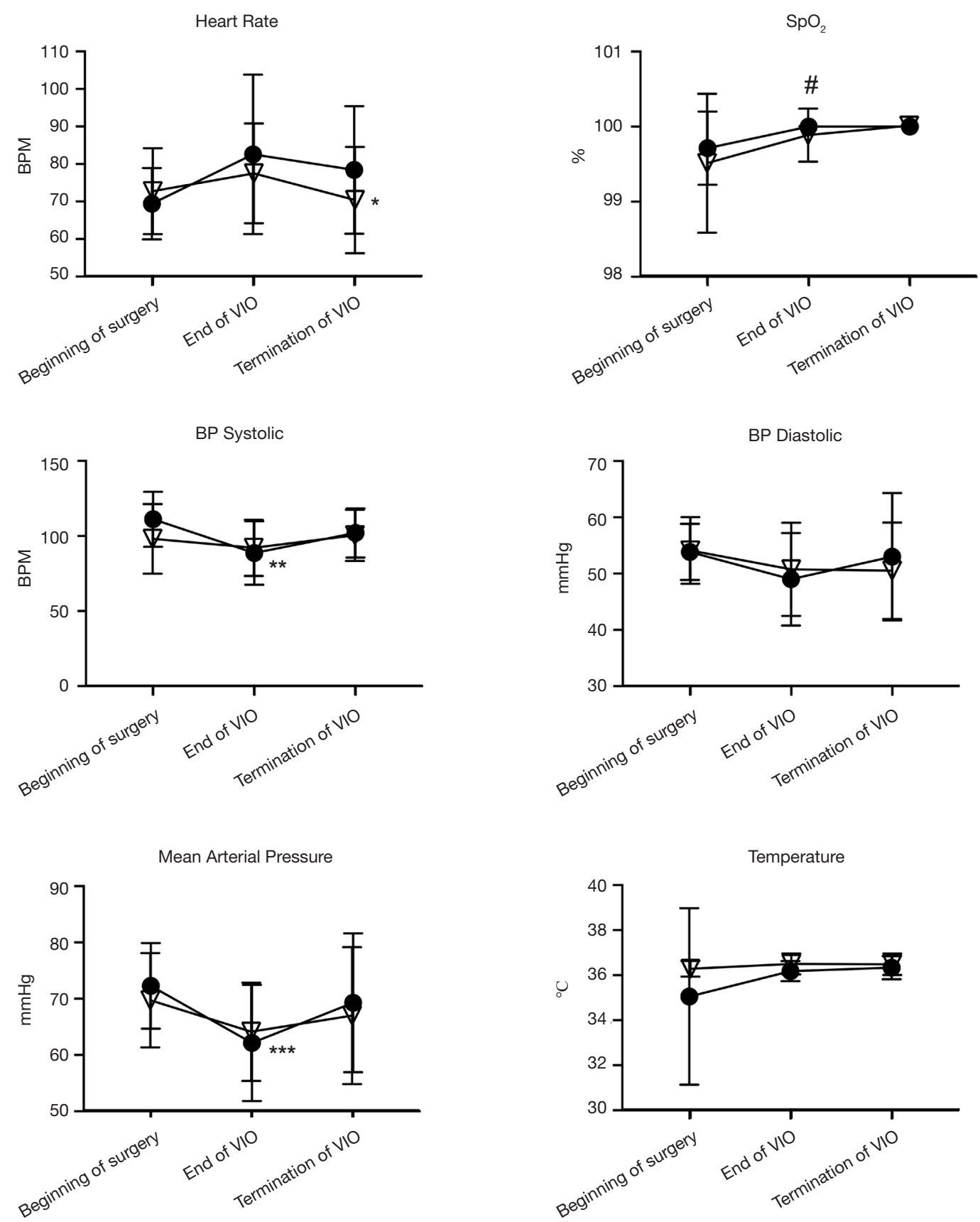

Figure 1 Systemic parameters of the full responders $(\bullet)$ and partial responder $(\nabla)$. Data represented as the mean and standard deviation. The observed completed cessation of hepatic microvascular blood flow (i.e., the full responders) at the end of VIO, was accompanied by a significant decrease in the systolic blood pressure and mean arterial pressure. Although no significant differences were observed in the full responders between any timepoints. In the partial responders, termination of VIO was accompanied by a significant decrease in the heart rate, while no other significant differences systemic responses were detected between any timepoints. ${ }^{*}=$ within group one-way ANOVA analysis; *, $\mathrm{P}<0.002$ vs. end of $\mathrm{VIO} ;{ }^{* *}, \mathrm{P}<0.006$ vs. beginning of surgery; ${ }^{* *}, \mathrm{P}<0.04$ vs. beginning of surgery. ${ }^{*}=$ between group unpaired $t$-test: \#, $\mathrm{P}<0.05$. Full responders vs. partial responders. BP, blood pressure; BPM, beats per minute; $\mathrm{SpO}_{2}$, peripheral capillary oxygen saturation; VIO, vascular inflow occlusion. 
A

A Functional Capillary Density
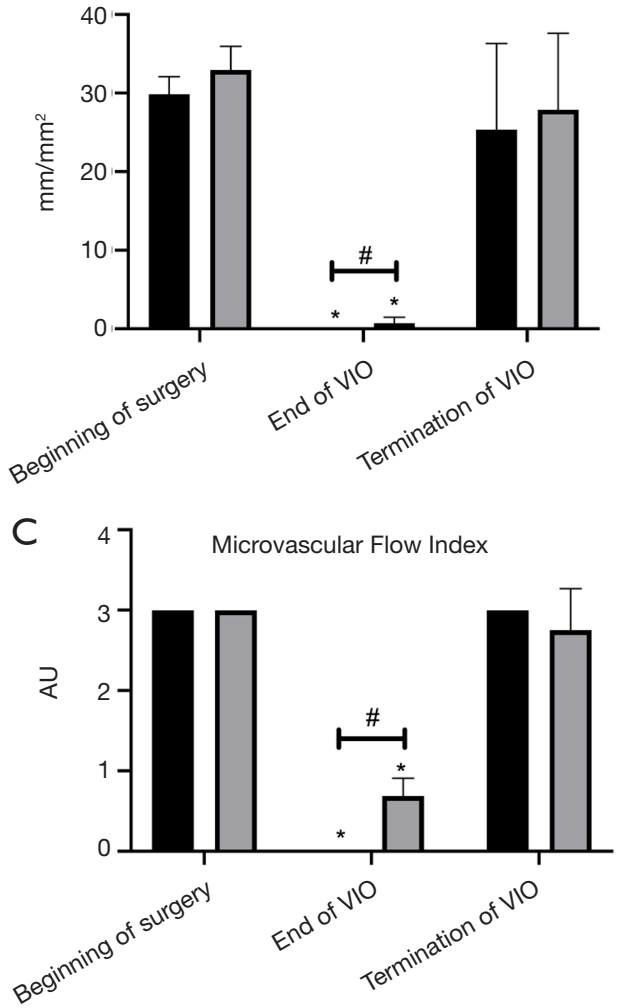

Full Responders

Partial Responders
B Sinusoidal Diameter
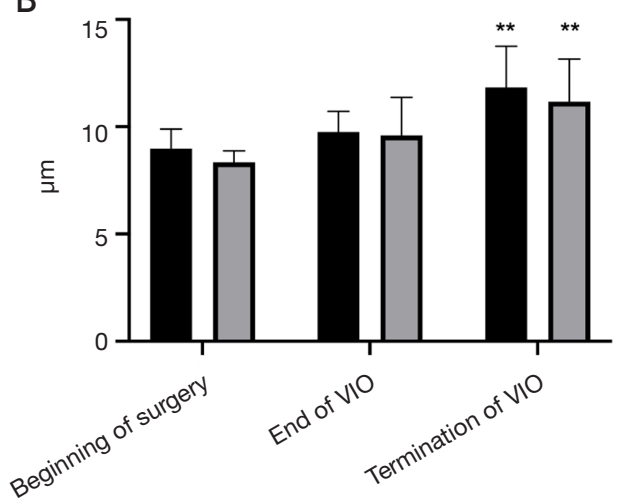

D Percentage of perfused vessel surface

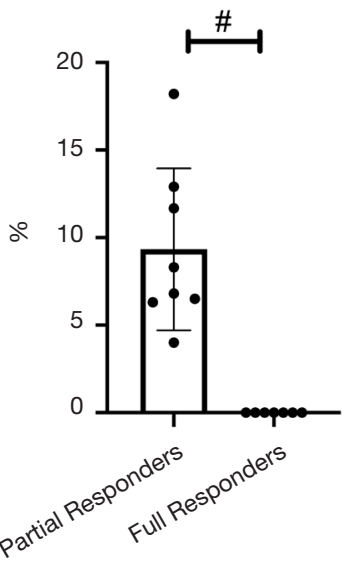

Figure 2 Hepatic microcirculatory parameters governing convection and flow. All data displayed as mean and standard deviation shown at the beginning of surgery, the end of VIO (20 minutes occlusion time) and within 1-minute following termination of VIO. (A) Functional capillary density. During assessment of the hepatic microcirculation at the end of VIO, perfused micro vessels (i.e., vessels with observable RBC flow) was seen in 8/15 patients; (B) sinusoidal diameter (SinD). The termination of VIO caused a significant increase in the SinD in both full and partial responders; (C) microvascular flow index (MFI). VIO caused a complete cessation of microvascular blood flow in the full responders. However, phenotypes of sluggish, intermittent and continuous blood flow were observed in the partial responders; (D) Percentage of perfused vessel surface. The partial responders showed residual blood flow of up to $10 \%$ in the field of view of the analysis screen, whereas the full responders showed complete cessation of microvascular blood flow. * = within group one-way ANOVA analysis; *, $\mathrm{P} \leq 0.02 v s$. beginning of surgery and termination of $\mathrm{VIO} ;{ }^{* *}, \mathrm{P}<0.02 v s$. Beginning of surgery. ${ }^{\#}=$ between group unpaired $t$-test: ${ }^{*}, \mathrm{P}<0.03$ full responders vs. partial responders. AU, arbitrary units; VIO, vascular inflow occlusion.

respectively). Following surgery, no patients sustained PHLF. Post-operatively, ALP levels were higher in the partial-responders when compared to the full-responders' group at post-operative day (POD) 1 (248.25 \pm 181.71 vs. $86.80 \pm 24.83 \mathrm{IU} / \mathrm{L}, \mathrm{P}<0.02$, respectively) and

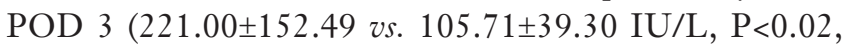
respectively). GGT levels at POD 5 were higher in the partial-responders group when compared to the fullresponders group $(387.3 \pm 280.8$ vs. $190.4 \pm 49.21, \mathrm{P}<0.01$, respectively).

Elevations in liver enzymes, AST and ALT, were mostly observed in the partial responders (Figure 3). A typical peak in AST and ALT levels was observed at POD 1 due to the surgical trauma. However, these values subsequently 

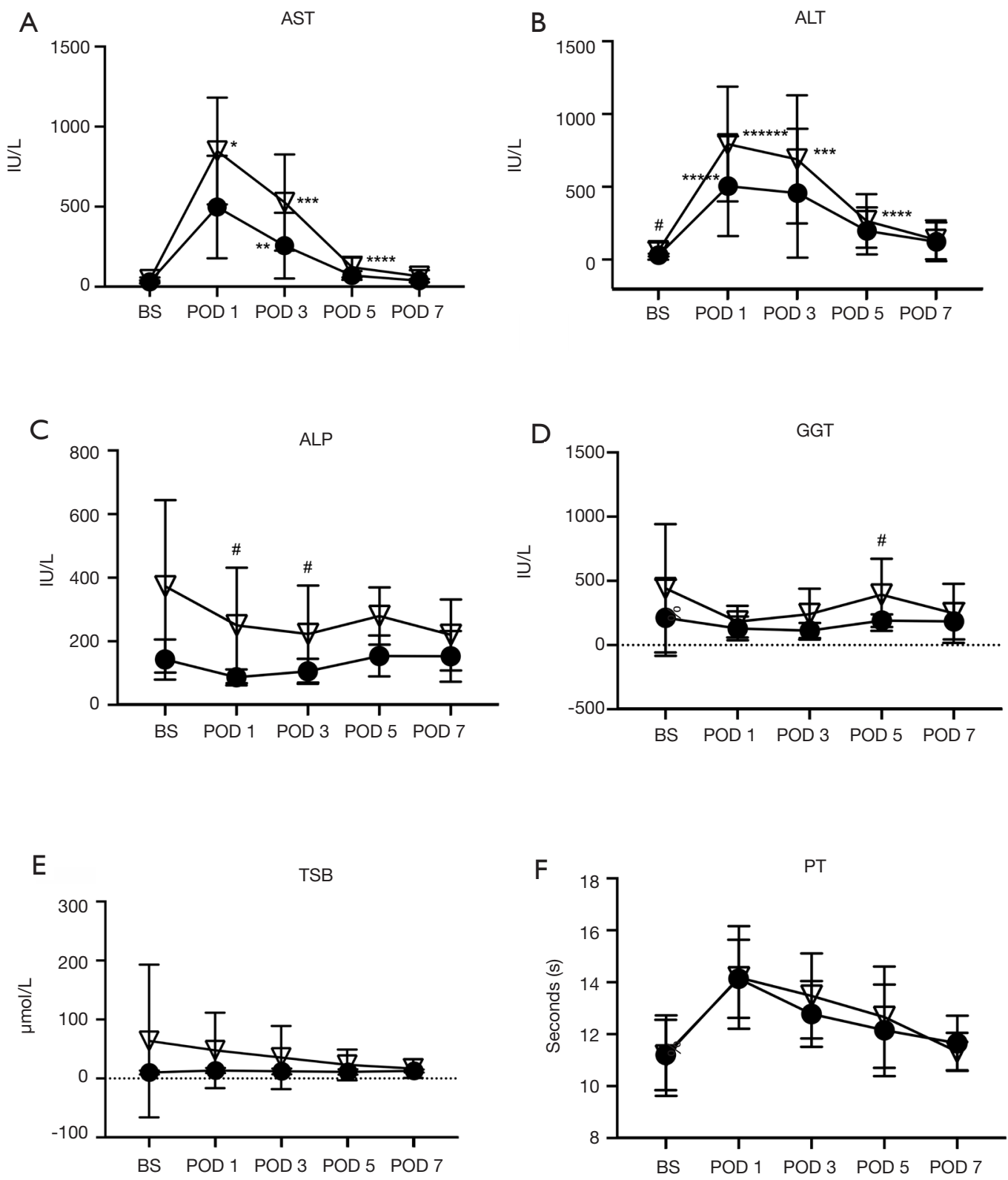

Figure 3 Liver function tests of the full responders $(-)$ and partial responder $(\nabla)$ shown at timepoints: before surgery (BS), post-operative day (POD) 1, POD 3, POD 5 and POD 7. All values are displayed as the mean and standard deviation. (A) Aspartate aminotransferase (AST); (B) alanine aminotransferase (ALT); (C) alkaline phosphatase (ALP); (D) gamma-glutamyl transferase (GGT); (E) total serum bilirubin (TSB); (F) prothrombin time $(\mathrm{PT}) .{ }^{*}=$ within group one-way ANOVA analysis: * $\mathrm{P}<0.05$ vs. BS, POD 3, POD 5 and $\mathrm{POD} 7$; **, $\mathrm{P}<0.05$ vs. $\mathrm{POD} 1$; ***, $\mathrm{P}<0.05$ vs. POD 5, and POD 7; ****, $\mathrm{P}<0.05$ vs. POD 7; ****, $\mathrm{P}<0.05$ vs. POD 5; *****, $\mathrm{P}<0.05$ vs. BS, POD 5 and POD $7 .{ }^{*}=$ between group unpaired $t$-test: ${ }^{*}, \mathrm{P}<0.05$ full responders $v s$. partial responders. 
returned back to normal values at POD 7. No other significant differences were observed within groups.

\section{Discussion}

The current study reported for the first-time a heterogeneity in the hepatic microvascular blood flow response to the application of VIO (Pringle manoeuvre) in humans using IDF imaging technique. In more than half of the patients, residual flow in the hepatic microcirculation was still observed, despite state-of-the-art application of VIO using a tourniquet around the hepatoduodenal ligament. In spite of this, no significant differences were observed in blood loss during the operation. Additionally, other observations such as arterial blood gases, blood pressure measurements and liver function enzymes were also uneventful. We hypothesize two possible explanations for the heterogeneity in microvascular perfusion observed during VIO: (I) back flow of blood from the hepatic venous system, or (II) presence of portal-systemic collateral systems and arterial collateral systems to the liver providing an alternative route for persistent microvascular blood flow

The presence of back flow from the systemic venous system during occlusion was demonstrated in experimental settings comparing hepatic microvascular perfusion parameters during inflow occlusion and total vascular exclusion (TVE) $(29,30)$. TVE induced a significant decrease in perfusion when compared to hepatic inflow occlusion alone, suggesting retrograde perfusion from the open venous system $(29,30)$. Since most animals lack a portal-systemic collateral system, the persistence of perfusion may be explained by back-flow alone. Extrahepatic collateral arteries supplying the liver with blood flow have been previously documented under the context of hepatic artery occlusion (31-33). During temporary balloon occlusion of the proper hepatic artery, arterial supply was immediately evident in $96 \%$ of patients evaluated using CT arteriography (31). This supports the presence of extrahepatic arterial blood supply to the liver in some patients $(34,35)$. Hence, naturally occurring systemic collateral systems bypassing the hepatoduodenal ligament are present in humans and contribute to the sustained microvascular blood flow observed in the partial responders. In actual fact, the presence of collateral portal-systemic circulation in humans (36) is the main reason why VIO is better tolerated in humans when compared to experimental portal occlusion in animal models. As the presence of hepatic venous back-flow was comparable in both full and partial responders, the differences in microvascular perfusion are more likely to be determined by the presence of collateral circulation.

Postoperative levels of ALT, AST and ALP are closely related to the volume of liver resected (37). Thus, to eliminate bias created by differences in resected volume, only patients with major hepatectomies were included in the study. The two groups were comparable with respect to the number of segments resected during surgery, however, since more patients in the partial responders group underwent (extended) right hemi-hepatectomies, this may explain the significantly higher ALP levels at POD 1 and POD 3 when compared to the full responders. Post-operatively, tests determining liver function (TSB, PT) also showed no significant differences between groups and no patients sustained PHLF. It may be theorised that the persistence of hepatic microvascular blood flow may to some extent, exacerbate IRI upon VIO. Previous clinical studies have compared the hepatic ultrastructure in patients receiving intermittent VIO versus no VIO using histological samples of the liver. Minor sinusoidal swelling, preservation of the hepatic microcirculation and greater expression of proteins associated with intracellular protection and repair were observed in the VIO group when compared to the control group $(38,39)$. Therefore, it may be postulated that ischemia and application of VIO during liver resection result in higher expression of protective genes at the molecular level, thereby preserving liver function of the FRL through protection of the hepatic microcirculatory ultrastructure $(40,41)$. Sustained microvascular blood flow during occlusion, may therefore not induce additional protective mechanisms.

Several strategies have been devised to protect the liver from IRI or to increase tolerance of the FRL to ischemia. Pharmacological interventions targeting various levels of the inflammatory cascade, ischemic preconditioning (IP), remote ischemic preconditioning (RIPC), all have been studied both in experimental settings (mostly rodent models) and clinically. Systematic reviews of randomised clinical trains (RCTs) comparing different pharmacological interventions have concluded no evidence in their protective nature against IRI $(42,43)$. The efficacy of IP depends on many variables, such as patient's age, duration of ischemia and the use of continuous or intermittent occlusion $(25,37)$. The current study has demonstrated considerable heterogeneity in interpatient microvascular perfusion, providing a possible explanation why clinical strategies to protect the liver against IRI have produced inconsistent 
results.

Our study is not without limitations, the small sample size determines this as a proof-of-concept paper and should provide the basis for future larger studies. Firstly, flow probes were not used in the current study for confirmation of complete occlusion of flow through the hepatic artery and the portal vein. However, the tourniquet technique is a widely used, straightforward procedure and has consistently demonstrated reliable inflow occlusion. Also in experimental studies in a porcine experimental model, the tourniquet technique showed complete occlusion as assessed with ultrasound transit-time technology to monitor blood flow (44). This renders the odds of incomplete occlusion due to technical errors highly unlikely. Secondly, the presence of caval backflow cannot be completed disregarded, as microcirculation monitoring was only performed at the end of VIO following 20 minutes of occlusion rather than immediately following occlusion and CVP was not monitored in all patients. Since the volumes of intraoperative blood loss are comparable between the full responders and partial responders, the role of caval backflow does not seem to be significant (45). Finally, occlusion assessments were only performed during the first round of VIO application. For future studies, it may be interested to assess whether hepatic microvascular blood flow is persistent in the partial responders during further rounds of VIO. Additionally, measurements of intrahepatic lactate concentrations may provide additional insight on the impact of microvascular perfusion during occlusion on hyperlactemia following liver resections.

In conclusion, this is a proof-of-concept study using IDF imaging technique, which showed a heterogenous hepatic microvascular perfusion response upon VIO. This may explain why attempts to introduce therapeutic interventions against IRI have shown to be inconsistent. The results of this study suggest intraoperative IDF imaging of the hepatic microcirculation may add to our current understanding of individual responses to VIO.

\section{Acknowledgments}

The authors would like to thank Olcay Dilken for his assistance and support during the data analysis phase of the liver microcirculation data using AVA 3.2. They would also like to thank staff of the operating theaters for their participation and assistance during the microcirculatory measurements.

Funding: None.

\section{Footnote}

Conflicts of Interest: All authors have completed the ICMJE uniform disclosure form (available at https://hbsn.amegroups. com/article/view/10.21037/hbsn.2020.02.04/coif). CI developed the Sidestream Dark Field imaging device and is listed as an inventor on the patent which is commercialized by MicroVision Medical under a license from the Amsterdam UMC. He received no royalties nor benefits from this license. He has been a consultant for MicroVision Medical previously, however has not been involved with this company for over 6 years, and holds no shares or stocks. Braedius Medical is a company owned by a relative of CI, which designed and developed the CytocamIDF imaging device. CI hold no financial relationship with Braedius Medical, does no hold shares or receive speaker or consultancy fees from the company. CI runs an internet site (www.microcirculationacademy.org) which offers training services that are related to the clinical microcirculation. TM van Gulik serves as an unpaid editorial board member of Hepatobiliary Surgery and Nutrition. The other authors have no conflicts of interest to declare.

Ethical Statement: The authors are accountable for all aspects of the work in ensuring that questions related to the accuracy or integrity of any part of the work are appropriately investigated and resolved. Ethical consent was given by the ethical committee at the Amsterdam UMC (ethical committee reference number W17_259). All participants eligible for inclusion received full explanation of the protocol and provided informed consent at least 24 hours before surgery. The study was carried out in accordance with the Declaration of Helsinki.

Open Access Statement: This is an Open Access article distributed in accordance with the Creative Commons Attribution-NonCommercial-NoDerivs 4.0 International License (CC BY-NC-ND 4.0), which permits the noncommercial replication and distribution of the article with the strict proviso that no changes or edits are made and the original work is properly cited (including links to both the formal publication through the relevant DOI and the license). See: https://creativecommons.org/licenses/by-nc$\mathrm{nd} / 4.0 /$.

\section{References}

1. Yang T, Zhang J, Lu JH, et al. Risk factors influencing 
postoperative outcomes of major hepatic resection of hepatocellular carcinoma for patients with underlying liver diseases. World J Surg 2011;35:2073-82.

2. Aragon RJ, Solomon NL. Techniques of hepatic resection. J Gastrointest Oncol 2012;3:28-40.

3. Man K, Fan ST, Ng IOL, et al. Tolerance of the liver to intermittent Pringle maneuver in hepatectomy for liver tumors. Arch Surg 1999;134:533-9.

4. Torzilli G, Procopio F, Donadon M, et al. Safety of intermittent Pringle maneuver cumulative time exceeding 120 minutes in liver resection: a further step in favor of the "radical but conservative" policy. Ann Surg 2012;255:270-80.

5. van Riel WG, van Golen RF, Reiniers MJ, et al. How much ischemia can the liver tolerate during resection? Hepatobiliary Surg Nutr 2016;5:58-71.

6. Teoh NC. Hepatic ischemia reperfusion injury: Contemporary perspectives on pathogenic mechanisms and basis for hepatoprotection-the good, bad and deadly. J Gastroenterol Hepatol 2011;26 Suppl 1:180-7.

7. Lemasters JJ, Theruvath TP, Zhong Z, et al. Mitochondrial calcium and the permeability transition in cell death. Biochim Biophys Acta 2009;1787:1395-401.

8. Mendes-Braz M, Elias-Miró M, Jiménez-Castro MB, et al. The Current State of Knowledge of Hepatic IschemiaReperfusion Injury Based on Its Study in Experimental Models [Internet]. BioMed Research International. 2012 [cited 2019 Feb 3]. Available online: https://www.hindawi. com/journals/bmri/2012/298657/

9. Peralta C, Jiménez-Castro MB, Gracia-Sancho J. Hepatic ischemia and reperfusion injury: Effects on the liver sinusoidal milieu. J Hepatol 2013;59:1094-106.

10. Hu Q, Ren H, Ren J, et al. Released Mitochondrial DNA Following Intestinal Ischemia Reperfusion Induces the Inflammatory Response and Gut Barrier Dysfunction. Sci Rep 2018;8:7350.

11. Fan C, Zwacka RM, Engelhardt JF. Therapeutic approaches for ischemia/reperfusion injury in the liver. J Mol Med (Berl) 1999;77:577-92.

12. Schreckenbach T, Liese J, Bechstein WO, et al. Posthepatectomy Liver Failure. Dig Surg 2012;29:79-85.

13. Grice P. Liver failure after major hepatic resection, a persistent clinical conundrum. J Hepato-Gastroenterol [Internet]. 2018 Jun 11 [cited 2019 Feb 8];2(1). Available online: https://www.pulsus.com/abstract/liver-failureafter-major-hepatic-resection-a-persistent-clinicalconundrum-4668.html

14. Puhl G, Schaser KD, Pust D, et al. Initial hepatic microcirculation correlates with early graft function in human orthotopic liver transplantation. Liver Transpl 2005;11:555-63.

15. Puhl G, Schaser KD, Vollmar B, et al. Noninvasive in vivo analysis of the human hepatic microcirculation using orthogonal polorization spectral imaging. Transplantation 2003;75:756-61.

16. Davies T, Wythe S, O'Beirne J, et al. Review article: the role of the microcirculation in liver cirrhosis. Aliment Pharmacol Ther 2017;46:825-35.

17. Panaro F, Benedetti E, Pineton de Chambrun G, et al. Indocyanine green fluorescence angiography during liver and pancreas transplantation: a tool to integrate perfusion statement's evaluation. Hepatobiliary Surg Nutr 2018;7:161-6.

18. Böker KHW, Franzen A, Wrobel M, et al. Regulation of hepatic blood flow in patients with liver cirrhosis and after liver transplantation. Pathophysiology 2000;6:251-60.

19. Richter S, Sperling J, Kollmar O, et al. Laser Doppler flowmetry of hepatic microcirculation during Pringle's maneuver: determination of spatial and temporal liver tissue perfusion heterogeneity. Eur Surg Res 2010;44:152-8.

20. Uz Z, Ince C, Rassam F, et al. Assessment of hepatic microvascular flow and density in patients undergoing preoperative portal vein embolization. HPB (Oxford) 2019;21:187-94.

21. Pulitano C, Joseph D, Sandroussi C, et al. Postreperfusion microcirculatory derangements after liver transplantation: Relationship to hemodynamics, serum mediators, and outcome. Liver Transpl 2017;23:527-36.

22. Puhl G, Schaser KD, Pust D, et al. The delay of rearterialization after initial portal reperfusion in living donor liver transplantation significantly determines the development of microvascular graft dysfunction. J Hepatol 2004;41:299-306.

23. Rauchfuss F, Scheuerlein H, Ludewig S, et al. In vivo assessment of the hepatic microcirculation after mesenterico-portal bypass (REX-shunt) using orthogonal polarization spectral imaging. Liver Int 2010;30:1339-45.

24. Schleimer K, Stippel DL, Kasper HU, et al. Improved microcirculation of a liver graft by controlled portal vein arterialization. J Surg Res 2004;116:202-10.

25. Hoekstra LT, van Trigt JD, Reiniers MJ, et al. Vascular Occlusion or Not during Liver Resection: The Continuing Story. Dig Surg 2012;29:35-42.

26. Esper RJ, Vilariño JO, Machado RA, et al. Endothelial dysfunction in normal and abnormal glucose metabolism. 
Adv Cardiol 2008;45:17-43.

27. Dobbe JGG, Streekstra GJ, Atasever B, et al. Measurement of functional microcirculatory geometry and velocity distributions using automated image analysis. Med Biol Eng Comput 2008;46:659-70.

28. Ince C, Boerma EC, Cecconi M, et al. Second consensus on the assessment of sublingual microcirculation in critically ill patients: results from a task force of the European Society of Intensive Care Medicine. Intensive Care Med 2018;44:281-99.

29. Tatsuma T, Kim YI, Kai T, et al. Importance of hepatovenous back-perfusion for maintenance of liver viability during the Pringle manoeuvre. Br J Surg 1995;82:1071-5.

30. Kim YI, Ishii T, Aramaki M, et al. The Pringle maneuver induces only partial ischemia of the liver. Hepatogastroenterology 1995;42:169-71.

31. Takeuchi Y, Arai Y, Inaba Y, et al. Extrahepatic arterial supply to the liver: observation with a unified CT and angiography system during temporary balloon occlusion of the proper hepatic artery. Radiology 1998;209:121-8.

32. Kim HC, Chung JW, Lee W, et al. Recognizing Extrahepatic Collateral Vessels That Supply Hepatocellular Carcinoma to Avoid Complications of Transcatheter Arterial Chemoembolization. RadioGraphics 2005;25:S25-39.

33. Yang L, Zhang XM, Ren YJ, et al. The Features of Extrahepatic Collateral Arteries Related to Hepatic Artery Occlusion and Benefits in the Transarterial Management of Liver Tumors. Radiol Res Pract [Internet]. 2013 [cited 2020 Jan 7];2013. Available online: https://www.ncbi.nlm. nih.gov/pmc/articles/PMC3806412/

34. Bola KK. A study of variant hepatic arterial anatomy and its relevance in current surgical practice. Int J Anat Res 2015;3:947-53.

35. Dandekar U, Dandekar K, Chavan S. Right Hepatic Artery: A Cadaver Investigation and Its Clinical Significance. Anat Res Int [Internet]. 2015 [cited 2020 Jan 7];2015. Available online: https://www.ncbi.nlm.nih.gov/ pmc/articles/PMC4695647/

36. Gallego C, Velasco M, Marcuello P, et al. Congenital and Acquired Anomalies of the Portal Venous System. RadioGraphics 2002;22:141-59.

37. Clavien PA, Selzner M, Rüdiger HA, et al. A prospective randomized study in 100 consecutive patients undergoing major liver resection with versus without ischemic preconditioning. Ann Surg 2003;238:843-50; discussion 851-2.

38. Man K, Lo CM, Liu CL, et al. Effects of the intermittent Pringle manoeuvre on hepatic gene expression and ultrastructure in a randomized clinical study. Br J Surg 2003;90:183-9.

39. Man K, Liang TB, Lo CM, et al. Hepatic stress gene expression and ultrastructural features under intermittent Pringle manoeuvre. Hepatobiliary Pancreat Dis Int 2002;1:249-57.

40. Kanoria S, Robertson FP, Mehta NN, et al. Effect of Remote Ischaemic Preconditioning on Liver Injury in Patients Undergoing Major Hepatectomy for Colorectal Liver Metastasis: A Pilot Randomised Controlled Feasibility Trial. World J Surg 2017;41:1322-30.

41. Winbladh A, Björnsson B, Trulsson L, et al. Ischemic preconditioning prior to intermittent Pringle maneuver in liver resections. J Hepatobiliary Pancreat Sci 2012;19:159-70.

42. Abu-Amara M, Gurusamy KS, Hori S, et al. Pharmacological interventions versus no pharmacological intervention for ischaemia reperfusion injury in liver resection surgery performed under vascular control. Cochrane Database Syst Rev 2009;(4):CD007472.

43. Abu-Amara M, Gurusamy K, Hori S, et al. Systematic review of randomized controlled trials of pharmacological interventions to reduce ischaemia-reperfusion injury in elective liver resection with vascular occlusion. HPB 2010;12:4-14.

44. Heijnen BHM, Straatsburg IH, Gouma DJ, et al. Decrease in core liver temperature with $10^{\circ} \mathrm{C}$ by in situ hypothermic perfusion under total hepatic vascular exclusion reduces liver ischemia and reperfusion injury during partial hepatectomy in pigs. Surgery 2003;134:806-17.

45. van Gulik TM, Graaf $W$ de, Dinant S, et al. Vascular Occlusion Techniques during Liver Resection. Dig Surg 2007;24:274-81.

Cite this article as: Shen L, Uz Z, Verheij J, Veelo DP, Ince Y, Ince C, van Gulik TM. Interpatient heterogeneity in hepatic microvascular blood flow during vascular inflow occlusion (Pringle manoeuvre). HepatoBiliary Surg Nutr 2020;9(3):271-283. doi: $10.21037 /$ hbsn.2020.02.04 